\title{
CARACTERÍSTICAS BIOLÓGICAS DE ADULTOS DE PODISUS NIGRISPINUS E SUPPUTIUS CINCTICEPS (HEMIPTERA: PENTATOMIDAE) ALIMENTADOS COM THYRINTEINA ARNOBIA (LEPIDOPTERA: GEOMETRIDAE)
}

\section{BIOLOGICAL CHARACTERISTICS OF ADULTS OF PODISUS NIGRISPINUS AND SUPPUTIUS CINCTICEPS (HEMIPTERA: PENTATOMIDAE) FED ON THYRINTEINA ARNOBIA (LEPIDOPTERA: GEOMETRIDAE)}

\author{
Anderson Mathias Holtz ${ }^{1 *}$; José Cola Zanuncio ${ }^{2}$; Jeanne Scardini Marinho ${ }^{2}$; \\ Dirceu Pratissoli ${ }^{1}$; Ângelo Pallini ${ }^{2}$ e Célia de Jesus Pereira ${ }^{2}$
}

\begin{abstract}
RESUMO
O gênero Eucalyptus (mirtacea exótica proveniente da Austrália) é o mais utilizado para reflorestamento no Brasil. Porém, somente a partir de 1966 é que este setor tomou impulso no país. Assim, além de exótica, a eucaliptocultura é recente. Periodicamente são observados surtos de lepidópteros desfolhadores (Thyrinteina arnobia, por exemplo). No Brasil, os predadores Podisus nigrispinus e Supputius cincticeps vem sendo utilizados contra lepidópteros desfolhadores, porém não se têm dados sobre o estabelecimento desses predadores em campo. Sendo assim, o objetivo deste trabalho foi verificar os aspectos biológicos de adultos de P. nigrispinus e $S$. cincticeps sobre lagartas de T. arnobia provenientes de plantas de Eucalyptus urophylla. Comparando-se os aspectos biológicos das duas espécies de predadores, observa-se que o desempenho de P. nigrispinus sobre as lagartas de T. arnobia foi melhor do que o desempenho de $S$. cincticeps. Este resultado demonstra que $P$. nigrispinus está conseguindo quebrar possíveis sistemas de defesa dessa espécie de herbívoro, tais como os compostos secundários que o herbívoro provavelmente estaria sequiestrando das plantas de eucalipto e, se os inimigos naturais (S. cincticeps, por exemplo) não estiverem adaptados a esses compostos, a sua performance no controle da praga e no seu próprio desenvolvimento podem ser afetados negativamente.
\end{abstract}

Palavras chave: Controle biológico, P. nigrispinus, S. cincticeps, eucalipto, aspectos biológicos.

\begin{abstract}
The Eucalyptus genus (an exotic mirtacea proceeding from Australia) is used for reforestation in Brazil. However, only after 1966 did this sector take impulse in the country. Thus, beyond exotic, the cultivation of this genus is recent. Defoliation caused by epidemics of lepidopterous species, such as Thyrinteina arnobia, for example, had been observed periodically. In Brazil Podisus nigrispinus and Supputius cincticeps are being used as predators against defoliator lepidopterous species, however in current literature information is not available about the establishment of these predators in the field. The objective of this work was to analyze the biological aspects of $\mathrm{P}$. nigrispinus and $\mathrm{S}$. cincticeps adults on $\mathrm{T}$. arnobia caterpillars collected on Eucalyptus urophylla plants. Comparing the biological aspects of the two predators species, the performance of $\mathrm{P}$. nigrispinus on the caterpillars of $\mathrm{T}$. arnobia was observed as better than the performance of $\mathrm{S}$. cincticeps. Based on these results it is possible to demonstrate that $\mathrm{P}$. nigrispinus is breaking possible systems of defense of this specie of herbivore, such as the secondary metabolites that the herbivore probably would be kidnapping from eucalyptus plants. If the natural enemies (S. cincticeps, for example) will not be adapt to these composites, its performance in the control of the pest and on its development can be negatively affected.
\end{abstract}

Key words: Biological control, P. nigrispinus, S. cincticeps, eucalyptus, biological aspects.

\footnotetext{
1 Universidade Federal do Espírito Santo, Centro de Ciências Agrárias, Departamento de Fitotecnia, Laboratório de Entomologia, Alto Universitário, Caixa Postal 16, 29500-000, Alegre, ES, E-mail: aholtz@insecta.ufv.br, pratissoli@ cca.ufes.br

2 Universidade Federal de Viçosa, Departamento de Biologia Animal, Setor de Entomologia, 36571-000, Viçosa, MG, E-mail: zanuncio@ufv.br, jeanne@insecta.ufv.br, pallini@ufv.br celia@insecta.ufv.br

* Autor para Correspondência
}

Fecha de Recepción: 01 Febrero 2006

Fecha de Aceptación: 07 Abril 2006 


\section{INTRODUÇÃO}

O gênero Eucalyptus (mirtacea exótica proveniente da Austrália) é a espécie mais utilizada para reflorestamento no Brasil (Zanuncio et al., 1993). Entretanto, somente a partir de 1966, com a lei de incentivos fiscais para reflorestamento, é que este setor começou a tomar impulso no país (Zanuncio et al., 1995; Santos et al., 2000). Assim, além de exótica, a eucaliptocultura é relativamente recente no Brasil em termos de adaptação de um novo gênero ou espécie em um novo ambiente.

No contexto entomológico e devido à presença de altas concentrações de compostos secundários existentes nas plantas de eucalipto, os pesquisadores acreditavam que insetos não causariam sérios danos a esta espécie de planta. Entretanto, observa-se o contrário. Há relatos de muitos insetos das ordens Lepidoptera, Coleoptera e Isoptera de mirtaceas nativas como jaboticabeira (Myrciaria cauliflora) e goiabeira (Psidium guajava) danificando plantas de eucalipto (Anjos et al., 1986; Zanuncio et al., 1990a,b e 1994a; Bragança et al., 1998).

Somente da ordem Lepidoptera já foram encontradas mais de 280 espécies nativas alimentando-se de plantas de eucalipto no Brasil, como Eupseudosoma aberrans e E. involuta (Arctiidae), Automeris spp., Eacles imperialis e Hylesia spp. (Saturniidae), Oxydia vesulia, Sabulodes caberata e Thyrinteina arnobia (Geometridae) (Otero, 1974; Anjos et al., 1987; Santos et al., 2000).

No Brasil, muitos trabalhos têm enfatizado o possível uso de Pentatomidae predadores, tais como Podisus nigrispinus, Supputius cincticeps, Brontocoris tabidus, entre outras espécies, no controle de lagartas desfolhadoras em plantios de eucalipto, pela facilidade de criação massal e eficiência em condições de laboratório (Zanuncio et al., 1994b). Os insetos são criados e liberados em áreas de reflorestamento, porém não se têm dados de estabelecimento desses predadores em campo.

A adaptação de herbívoros em um novo hospedeiro tem sido alvo de estudos ecológicos há algumas décadas (Agrawal, 2000). Consideravelmente menor atenção tem sido dada à investigação sobre como predadores se adaptam a novas plantas hospedeiras. Desta forma, o objetivo do trabalho foi estudar os aspectos biológicos de P. nigrispinus e $S$. cincticeps (Hemiptera: Pentatomidae) sobre lagartas de T. arnobia (Lepidoptera: Geometridae).
Com os resultados obtidos poderemos determinar qual a espécie de predador está mais adaptada a essa espécie de herbívoro.

\section{MATERIAL E MÉTODOS}

\section{INSTALAÇÕES, CRIAÇÃO E MANUTENÇÃO DE T. ARNOBIA}

Os adultos de T. arnobia, provenientes de coleta em campo em plantios de eucalipto no Estado de Minas Gerais, foram levados para laboratório de Acarologia da Universidade Federal de Viçosa (UFV). Estes adultos foram mantidos em temperatura de $25 \pm 2^{\circ} \mathrm{C}$, fotofase de 12 horas e umidade relativa de $70 \pm 10 \%$. Casais de T. arnobia foram individualizados e colocados em potes plásticos $(500 \mathrm{ml})$ com tampa plástica, contendo uma abertura central, vedada com tela de malha fina, tipo organza. Nos potes, foram colocadas tiras de papel presas à tampa para que nelas fossem efetuadas as posturas. Logo após a eclosão dos ovos, as lagartas de T. arnobia foram colocadas em sacos constituídos por tecido tipo organza $(0,70 \times 0,40 \mathrm{~m})$ envolvendo galhos de plantas de eucalipto sem danos. Nos primeiros estádios, as lagartas foram removidas para outros galhos com sacos de organza quando as folhas começavam a secar. A troca de galhos foi mais freqüente a partir do quarto estádio, período em que as lagartas alimentam-se mais vorazmente. Quando as lagartas alcançaram a fase de pupa, as mesmas foram retiradas dos sacos de organza e colocadas em potes plásticos (conforme descrição anterior) e levados para o laboratório de Acarologia da UFV e mantidos sob as mesmas condições ambientais.

\section{CRIAÇÃO DE P. NIGRISPINUS E S. CINCTICEPS E DESENVOLVIMENTO DOS EXPERIMENTOS}

Os testes foram conduzidos no laboratório de Acarologia da UFV, em Viçosa, Minas Gerais, com P. nigrispinus e $S$. cincticeps, a $25 \pm 2^{\circ} \mathrm{C}$ de temperatura, $70 \pm 10 \%$ de U.R. e fotoperíodo de $12 \mathrm{~h}$, em lagartas de T. arnobia criadas em plantas de Eucalyptus urophylla.

Ninfas de primeiro estádio de $P$. nigrispinus e $S$. cincticeps foram obtidas da criação massal do laboratório de Entomologia Florestal do Departamento de Biologia Animal da UFV. Essas ninfas foram criadas, sob as mesmas condições 
laboratoriais, do primeiro estádio à fase adulta em grupos de 5 em placas de Petri $(15,0$ x 1,2 cm) contendo um chumaço de algodão no seu interior, sendo diariamente umedecido com água destilada. A alimentação desses insetos foi realizada seguindo-se a metodologia de Zanuncio et al. (1994b) em dieta com pupas de Tenebrio molitor L., 1758 (Coleoptera: Tenebrionidae).

No início da fase adulta, os percevejos das duas espécies de predadores foram pesados em balança com precisão de $0,1 \mathrm{mg}$, e sexados pela aparência externa da genitália e tamanho do corpo. No primeiro dia após passarem para a fase adulta, $P$. nigrispinus e $S$. cincticeps foram acasalados e distribuídos em placas de Petri (conforme descrição anterior) (um casal por placa). Os mesmos foram alimentados diariamente com lagartas de T. arnobia de $5^{\circ}$ ou $6^{\circ}$ instar, provenientes de eucalipto. Foram realizadas 25 repetições em cada tratamento.

Diariamente, foram observados o período de oviposição, o número de posturas e o número de ovos, além da longevidade de machos e fêmeas. As posturas foram retiradas das placas de Petri e colocadas em placas de Petri de 9,0 x 1,2cm para se observar à fertilidade dos ovos e o número de ninfas. A taxa de postura e a oviposição diária por fêmea foram calculadas dividindo-se o número de posturas e o número total de ovos pelo período reprodutivo, respectivamente.

Os resultados foram transformados em arcoseno, visando observar-se à homogeneidade de variância e a normalidade dos dados, respectivamente. Posteriormente, os dados foram submetidos ao teste
F a 5\% de significância. Os dados de longevidade foram usados no modelo de distribuição de Weibull, a fim de se estimar o modelo de sobrevivência de $P$. nigrispinus e $S$. cincticeps (Sgrillo, 1982).

\section{RESULTADOS}

Analisando-se os aspectos biológicos de P. nigrispinus e $S$. cincticeps sobre lagartas de T. arnobia, observa-se que houve diferença significativa $(\mathrm{P}<0,05)$ para os parâmetros observados entre essas espécies de predadores. Apenas no parâmetro de pós-oviposição não foi observada diferença significativa $(\mathrm{P}>0,05)$ (Tabela 1$)$.

O número de posturas foi maior para as fêmeas de $P$. nigrispinus $(2,44)$ quando comparado às fêmeas de $S$. cincticeps $(1,50)$ (Figura 1). O número de ovos também foi maior para as fêmeas de $P$. nigrispinus alimentadas com lagartas de T. arnobia $(57,00)$ em relação a fêmeas de $S$. cincticeps $(30,17)$ (Figura 2). Esta mesma tendência ocorreu para o número de ovos/postura. Fêmeas de $P$. nigrispinus apresentaram um maior número de ovos/postura $(16,32)$ quando comparado a fêmeas de $S$. cincticeps $(5,46)$ (Tabela 1).

O número de ovos férteis também foi maior $(\mathrm{P}<0,05)$ em fêmeas de $P$. nigrispinus $(52,36)$ se comparado às fêmeas de $S$. cincticeps $(20,67)$ (Tabela 1). Conseqüentemente, observou-se um maior número de ovos viáveis para fêmeas de $P$. nigrispinus $(51,00)$ quando comparado a fêmeas de $S$. cincticeps $(18,67)$ (Figura 3 ).

Tabela 1

Aspectos biológicos de adultos de Podisus nigrispinus e Supputius cincticeps (Heteroptera: Pentatomidae) criados sobre lagartas de Thyrinteina arnobia (Lepidoptera: Geometridae)

\begin{tabular}{|l|c|c|}
\hline \multicolumn{1}{|c|}{ Parâmetros } & Podisus nigrispinus & Supputius cincticeps \\
\hline Número de Ovos/Postura & $16,32 \pm 13,87 \mathrm{a}$ & $6,28 \pm 10,48 \mathrm{~b}$ \\
Número de Ovos Férteis & $52,36 \pm 41,49 \mathrm{a}$ & $20,67 \pm 11,54 \mathrm{~b}$ \\
Taxa de Oviposição & $0,53 \pm 0,46 \mathrm{a}$ & $0,19 \pm 0,38 \mathrm{~b}$ \\
Oviposição Diária & $13,51 \pm 14,06 \mathrm{a}$ & $4,22 \pm 9,02 \mathrm{~b}$ \\
Pré-Oviposição & $6,28 \pm 5,90 \mathrm{a}$ & $2,96 \pm 5,53 \mathrm{~b}$ \\
Oviposição & $4,56 \pm 4,90 \mathrm{a}$ & $3,16 \pm 4,02 \mathrm{~b}$ \\
Pós-Oviposição & $5,93 \pm 4,89 \mathrm{a}$ & $5,17 \pm 3,02 \mathrm{a}$ \\
\hline
\end{tabular}

Médias seguidas de, pelo menos, uma mesma letra nas linhas, não diferem entre si, pelo teste F, ao nível de 5\% de significância. 


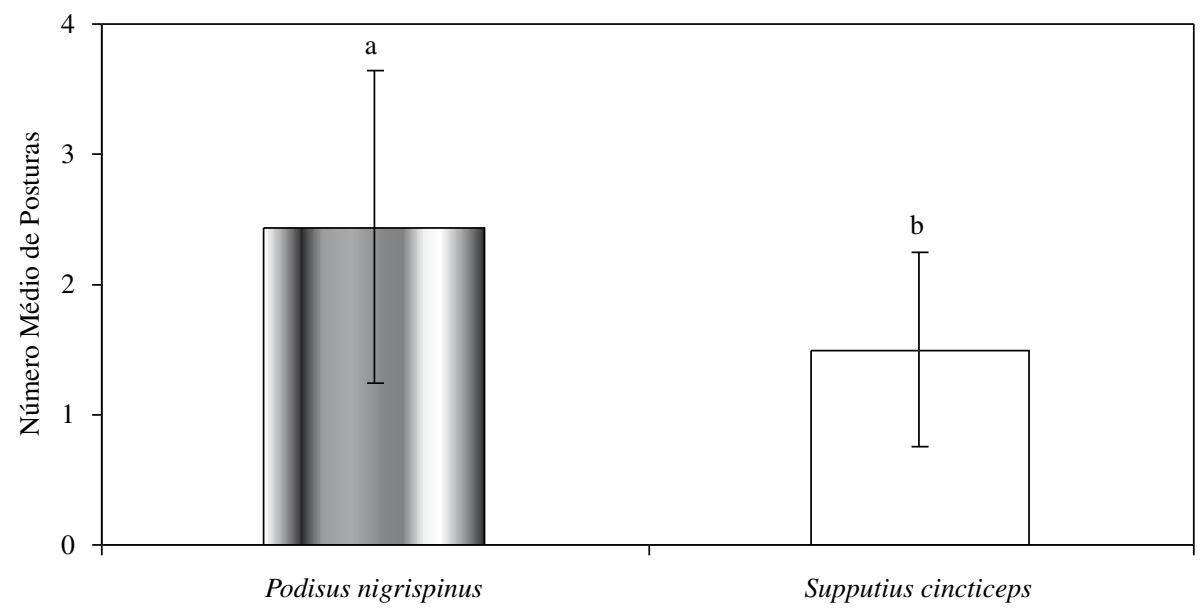

Figura 1. Número de posturas de Podisus nigrispinus e Supputius cincticeps (Heteroptera: Pentatomidae) criados sobre lagartas de Thyrinteina arnobia (Lepidoptera: Geometridae). Médias com letras diferentes diferem entre si, pelo teste F, a 5\% de significância.

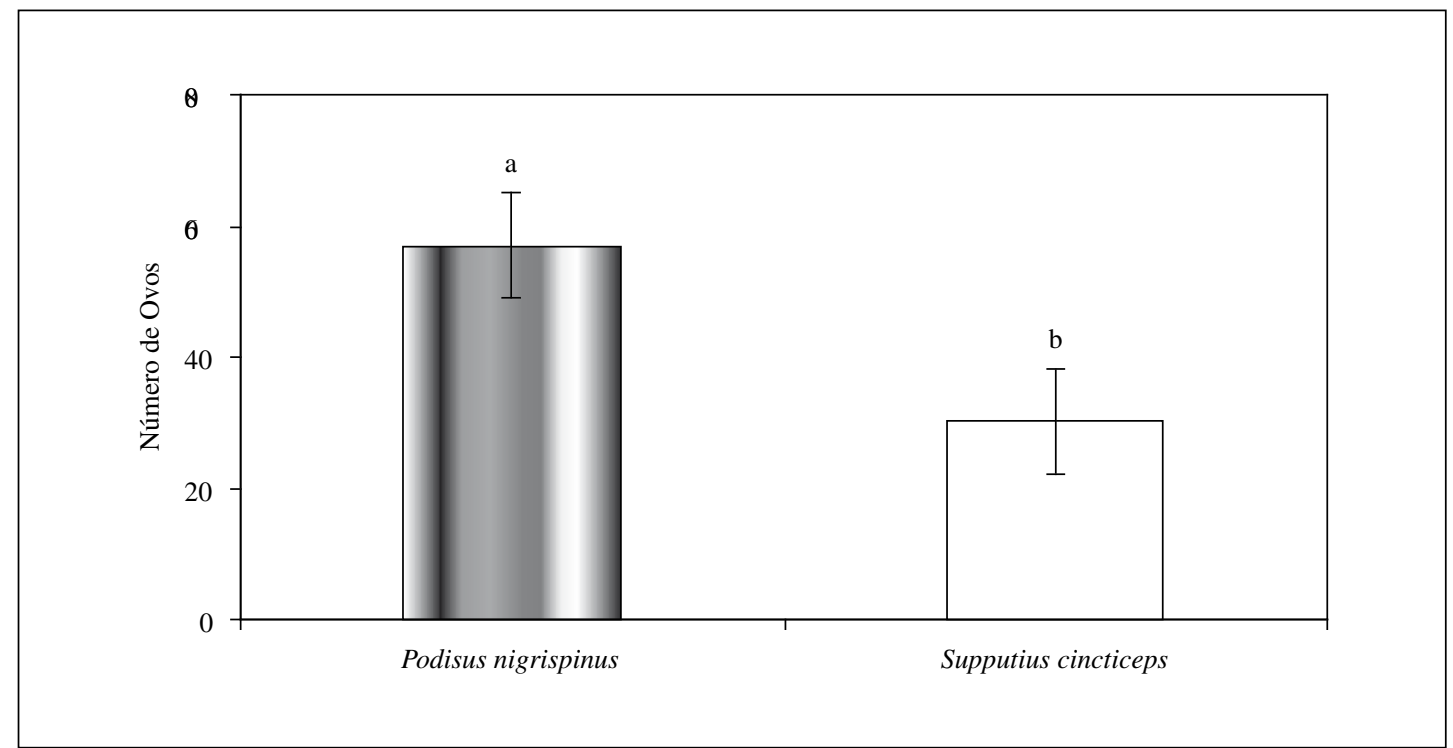

Figura 2. Número médio de ovos de Podisus nigrispinus e Supputius cincticeps (Heteroptera: Pentatomidae) criados sobre lagartas de Thyrinteina arnobia (Lepidoptera: Geometridae). Médias com letras diferentes diferem entre si, pelo teste F, a 5\% de significância.

A taxa de oviposição e a oviposição diária também foram maiores $(\mathrm{P}<0,05)$ em fêmeas de P. nigrispinus quando comparado a fêmeas de $S$. cincticeps, sendo de 0,53 e 0,19 para taxa de oviposição e de 13,51 e 4,22 para oviposição diária de fêmeas de $P$. nigrispinus e S. cincticeps, respectivamente (Tabela 1).
A pré-oviposição, bem como a oviposição também foram maiores $(\mathrm{P}<0,05)$ em fêmeas de $P$. nigrispinus quando comparado a fêmeas de $S$. cincticeps. A pré-oviposição foi de 6,28 dias para fêmeas de $P$. nigrispinus contra 2,96 dias para fêmeas de S. cincticeps (Tabela 1). A oviposição foi de 4,56 


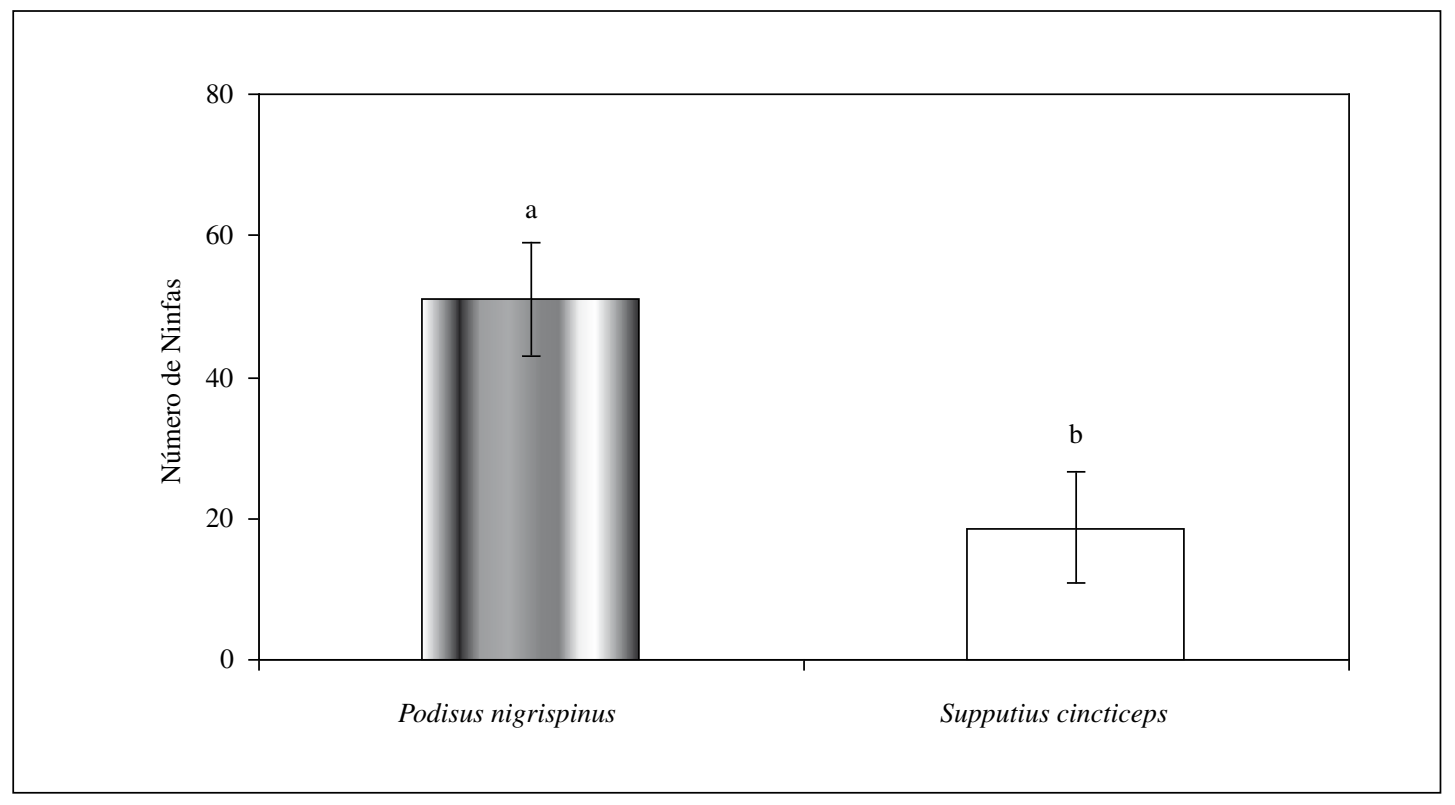

Figura 3. Número médio de ovos viáveis de Podisus nigrispinus e Supputius cincticeps (Heteroptera: Pentatomidae) criados sobre lagartas de Thyrinteina arnobia (Lepidoptera: Geometridae). Médias com letras diferentes diferem entre si, pelo teste F, a 5\% de significância.

dias para fêmeas de P. nigrispinus contra 3,16 dias para as fêmeas de $S$. cincticeps (Tabela 1).

Analisando-se a curva de sobrevivência de adultos de P. nigrispinus (Figura 4), nota-se que em torno de sete dias tinha-se uma sobrevivência aproximada de $85 \%$ de indivíduos desse predador alimentados com lagartas de T. arnobia, enquanto que no mesmo período, a porcentagem de sobrevivência de $S$. cincticeps estava em torno de 50\% (Figura 4). Em todo momento, a curva de sobrevivência de $P$. nigrispinus ficou acima da curva de sobrevivência de $S$. cincticeps (Figura 4), sendo que os indivíduos de P. nigrispinus sobreviveram em torno de 28 dias, enquanto que indivíduos de $S$. cincticeps sobreviveram em torno de 22 dias (Figura 4).

\section{DISCUSSÃO}

O predador P. nigrispinus apresentou melhor desempenho (maior taxa de sobrevivência, maior número de ovos por postura, maior número de ovos férteis, maior taxa de oviposição e oviposição diária) quando criado sobre lagartas de T. arnobia do que o predador $S$. cincticeps. Provavelmente, P. nigrispinus está mais adaptada aos possíveis compostos seqüestrados pelo herbívoro do que esta espécie de predador. Segundo Coll \& Guershon
(2002) e Vlieger et al. (2004) os herbívoros, como T. arnobia, não são apenas passíveis aos sistemas de defesa das plantas, muitos desses insetos herbívoros conseguem se especializar em plantas com compostos secundários (taninos, fenóis, etc.), e também, em seqüestrar e utilizar esses compostos, se tornando impalatáveis e/ou igualmente tóxicos aos inimigos naturais.

Um exemplo de seqüestro de compostos por herbívoro foi demonstrado por Holtz et al. (2005). Estes autores observaram que quando $P$. nigrispinus foi alimentado com T. molitor (alimentação a base de farelo de trigo e chuchu), o seu desempenho foi melhor quando comparado ao mesmo predador alimentado com lagarta de T. arnobia. Provavelmente, esse resultado está relacionado com o fato de que as lagartas de T. arnobia se alimentaram de folhas de eucalipto, que são ricas em compostos secundários. Segundo Sznajder \& Harvey (2003) o efeito das plantas na qualidade da presa e/ou hospedeiro, e subseqüentemente no desenvolvimento de inimigos naturais, pode ser devido à presença de aleloquímicos no tecido hospedeiro (via seqüestro). Schenk \& Bacher (2002) e Müller \& Brakefield (2003) argumentam que insetos herbívoros que não apresentarem proteção química podem ser altamente afetados pelos seus inimigos naturais. 


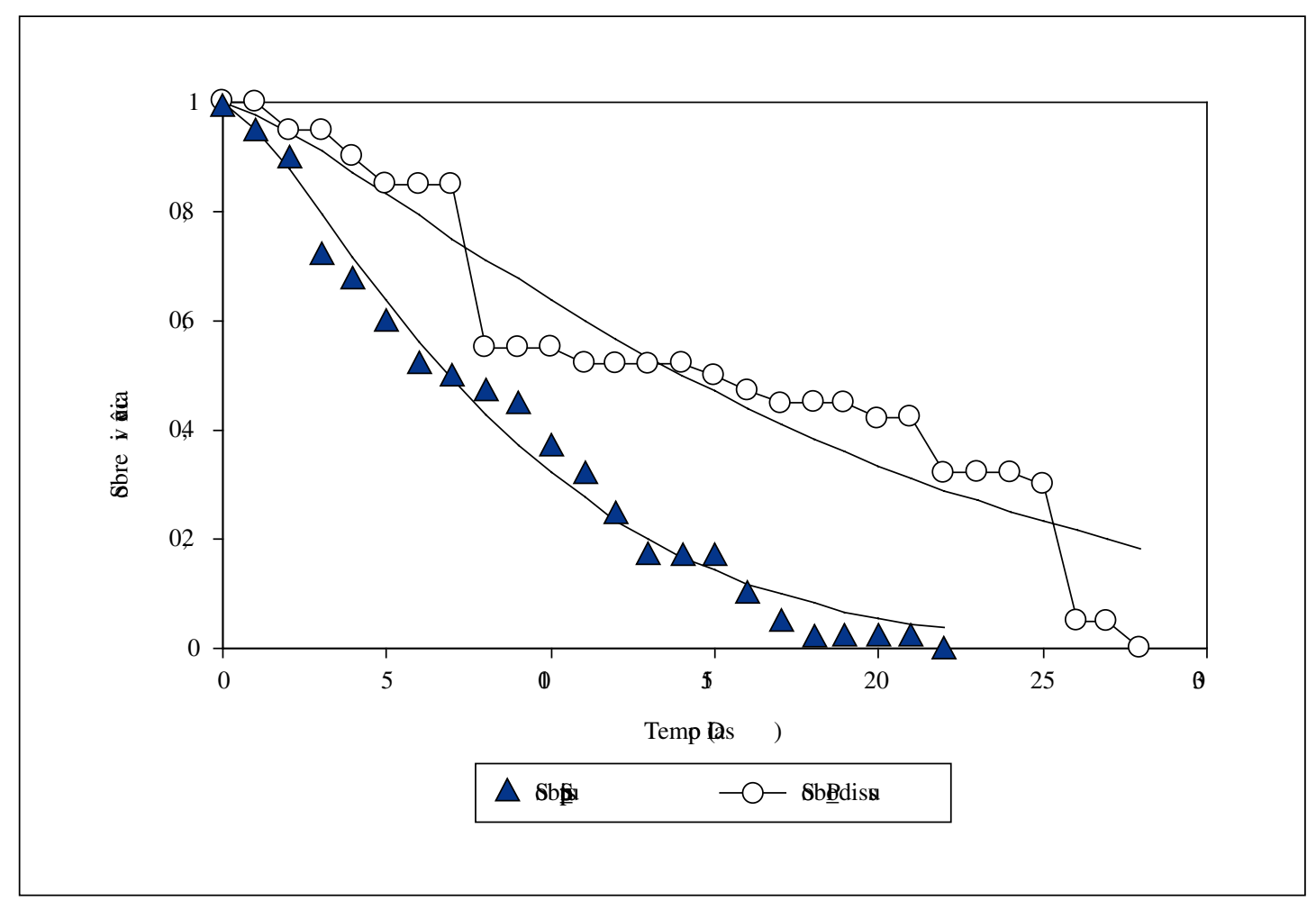

Figura 4. Curva de sobrevivência (distribuição de Weibull) de adultos de Podisus nigrispinus e Supputius cincticeps (Heteroptera: Pentatomidae) criados sobre lagartas de Thyrinteina arnobia (Lepidoptera: Geometridae.

Desta forma, herbívoros que se alimentam de folhas de eucalipto poderiam estar seqüestrando os compostos tóxicos provenientes destas plantas (pois as mesmas têm altas concentrações de compostos secundários) e tornando-se impalatáveis ou tóxicas aos inimigos naturais, o que provavelmente ocorreu neste experimento com ambos os predadores alimentados com as lagartas de T. arnobia. Contudo, $P$. nigrispinus se mostrou mais adaptado aos possíveis compostos seqüestrados por esse herbívoro do que S. cincticeps.

De Clercq \& Degheele (1992), Wiedenmann et al. (1996) e De Clercq et al. (2000) argumentam que aleloquímicos de plantas podem influenciar a performance de predadores, como os heterópteros, diretamente, pois tais predadores utilizam as plantas para a retirada de nutrientes e água, ou indiretamente, através de suas presas, que seqüestram compostos secundários, e, se os mesmos não estiverem adaptados àquela espécie de planta, podem ter seu desempenho afetado negativamente. Weiser \& Stamp (1998) mostraram que adicionando aleloquímicos, retirados de plantas de tomate, em uma dieta artificial fornecida para Manduca sexta L., causou efeito negativo sobre a performance e desenvolvimento do predador Podisus maculiventris. Estes mesmos autores argumentam que a sobrevivência no $5^{\circ}$ instar desse predador foi afetada quando lhe foi oferecida lagartas de M. sexta criadas com a dieta onde foram adicionados os aleloquímicos provenientes de tomate. No trabalho realizado por Montillor et al. (1991) foi observado que a vespa Mischocyttarus flavitarsis foi afetada negativamente por alcalóides que foram seqüestrados pelas lagartas de suas plantas hospedeiras.

T. arnobia é considerado o principal lepidóptero praga em plantios de eucalipto, devido aos seus surtos periódicos e danos causados aos plantios dessa essência florestal (Santos et al., 1996). Em trabalhos realizados por Holtz et al. (2003a, b), observou-se que o desempenho desse herbívoro foi melhor em plantas de eucalipto do que em plantas de goiaba. Desta forma, observa-se que T. arnobia está mais especializada em plantas de eucalipto do que em plantas de goiaba. Dobler (2001) comenta que uma óbvia pré-condição para o seqüestro de compostos 
secundários de plantas está na freqüência que determinado herbívoro se alimenta da planta. Insetos herbívoros especializados em uma determinada planta são mais passíveis de seqüestrarem compostos que insetos herbívoros generalistas, que se alimentam de diferentes espécies de plantas (Dobler, 2001). Os insetos herbívoros especialistas são conhecidos por exibirem adaptações aos compostos secundários e até mesmo em utilizarem esses compostos como estimulantes na sua alimentação (Renwick \& Lopez, 1999; Roessingh et al., 2000; Stadler, 2000).

Segundo Nishida (2002) e Sznajder \& Harvey (2003) uma vez ingerido pelos insetos herbívoros, a substância seqüestrada é reabsorvida através da membrana intestinal, transportada para a hemolinfa e depositada em diversas partes do corpo e, desta forma, tornando os mesmos tóxicos ou impalatáveis a seus inimigos naturais (Wink et al., 2000; Müller et al., 2001; Harvey et al., 2003).

Vários estudos têm relatado que aleloquímicos em presas e/ou hospedeiros afetam negativamente o crescimento, desenvolvimento, sobrevivência e morfologia de predadores e parasitóides (Duffey et al., 1986; Gunasena et al., 1990; Paradise \& Stampe, 1993; Havill \& Raffa, 2000). Contudo, em muitos casos estes efeitos são mais freqüentes em inimigos naturais generalistas (como em herbívoros generalistas), enquanto que os inimigos naturais

\section{REFERÊNCIAS BIBLIOGRÁFICAS}

AGRAWAL, A. A. 2000. Specificity of induced resistance in wild radish: causes and consequences for two specialist and two generalist caterpillars. Oikos, 89: 493-500.

ANJOS, N.; SANTOS, G. P.; ZANUNCIO, J. C. 1986. Pragas do eucalipto e seu controle. Informe Agropecuário, 12: 50-58.

ANJOS, N.; SANTOS, G. P.; ZANUNCIO, J. C. 1987. A lagarta-parda, Thyrinteina arnobia Stoll, 1782 (Lepidoptera: Geometridae) desfolhadora de eucaliptos. EPAMIG, 56 p. Boletim Técnico, 25.

BARBOSA, P.; GROSS, P.; KEMPER, J. 1991. Influence of plant allelochemicals on the performance of the tobacco hornworm and its parasitoid Cotesia congregata. Ecology, 72: 1567-1575.

BRAGANCA, M. A. L.; ZANUNCIO, J. C.; PICANÇO, M.; LARANJEIRO, A. J. 1998. Effects of environmental heterogeneity on Lepidoptera and Hymenoptera populations in Eucalyptus plantations in Brazil. Forest Ecology and Management, 103: 287-292.

COLL, M.; GUERSHON, M. 2002. Omnivory in terrestrial arthropods: mixing plant and prey diets. Annual Review of Entomology, 47: 267-297. especialistas estão, aparentemente, mais adaptados às toxinas das plantas (Barbosa et al., 1991; Paradise \& Stamp, 1993; Vinson, 1999; Harvey et al., 2003). Desta forma, por ser $S$. cincticeps um predador generalista e com curta história co-evolutiva com plantas de eucalipto, estaria exposto aos compostos secundários dessa essência florestal.

Os resultados obtidos no presente trabalho permitem concluir que $S$. cincticeps foi afetado por lagartas de T. arnobia provenientes de eucalipto. Isto indica que este predador não estaria adaptado aos compostos secundários dessa essência florestal, em comparação com o predador $P$. nigrispinus. Desta forma, compostos de defesa da planta que inicialmente agiriam em benefício da própria planta estariam afetando negativamente populações de inimigos naturais, ainda mais se os mesmos não estiverem adaptados a esses compostos.

\section{AGRADECIMENTOS}

Ao Conselho Nacional de Desenvolvimento Científico e Tecnológico (CNPq), à Coordenação de Aperfeiçoamento de Pessoal de Nível Superior (CAPES) e à Fundação de Amparo a Pesquisa do Estado de Minas Gerais (FAPEMIG) pela concessão de bolsas.

OTERO, L.S. 1974. Contribuição ao conhecimento da entomofauna do Parque Florestal da Tijuca. Brasil Florestal, 5: 37-39.

DE CLERCQ, P.; DEGHEELE, D. 1992. Plant feeding by two species of predatory bugs of the genus Podisus (Heteroptera: Pentatomidae). Meded. Fac. Landbouww. Univ. Gent, 57: 591-596.

DE CLERCQ, P.; MOHAGHEGH, J.; TIRRY, L. 2000. Effect of host plant on the functional response of the predator Podisus nigrispinus (Heteroptera: Pentatomidae). Biological Control, 18: 65-70.

DOBLER, S. 2001. Evolutionary aspects of defense by recycled plant compounds in herbivorous insects. Basic and Applied Ecology, 2: 15-26.

DUFFEY, S. S.; BLOEM, K. A.; CAMPBELL, B. C. 1986. Consequences of sequestration of plant natural products in plant-insect-parasitoid interactions. In BOETHEL, D.J.; EIKENBARY, R.D. Interactions of plant resistance and parasitoids and predators of insects. Horwood, Chichester, UK, p. 31-60.

GUNASENA, G. H.; VINSON, S. B.; WILLIAMS, H. J. 1990. Effects of nicotine on growth, development and survival of the 
tobacco budworm (Lepidoptera: Noctuidae) and the parasitoid Campoletis sonorensis (Hymenoptera: Ichneumonidae). Journal of Economic Entomology, 83: 1777-1782.

HARVEY, J. A.; VAN DAM, N. M.; GOLS, R. 2003. Interactions over four trophic levels: foodplant quality affects development of a hyperparasitoid as mediated through a herbivore and its primary parasitoid. Journal of Animal Ecology, 72: 520-531

HAVILL, N. P.; RAFFA, K. F. 2000. Compound effects of induced plant responses on insect herbivores and parasitoids: implications for tritrophic interactions. Ecological Entomology, 25: 171-179.

HOLTZ, A. M.; OLIVEIRA, H. G.; PALLINI, A.; MARINHO, J. S.; ZANUNCIO, J. C.; OLIVEIRA, C. L. 2003a. Adaptação de Thyrinteina arnobia em novo hospedeiro e defesa induzida por herbívoros em eucalipto. Pesquisa Agropecuária Brasileira, 38: 453-458.

HOLTZ, A. M.; OLIVEIRA, H. G.; PALLINI, A.; VENZON, M.; ZANUNCIO, J. C.; OLIVEIRA, C. L.; MARINHO, J. S.; ROSADO, M. C. 2003b. Desempenho de Thyrinteina arnobia Stoll (Lepidoptera: Geometridae) em eucalipto e goiaba: o hospedeiro nativo não é um bom hospedeiro? Neotropical Entomology, 32: 427-431.

HOLTZ, A. M.; ZANUNCIO, J. C.; PALLINI, A.; OLIVEIRA, C. L.; MARINHO, J. S.; PEREIRA, C. J. Potencial reprodutivo e análise de sobrevivência de Podisus nigrispinus (Heteroptera: Pentatomidae) sobre duas presas: Thyrinteina arnobia (Lepidoptera: Geometridae) e Tenebrio molitor (Coleoptera: Tenebrionidae). 2005. (In Press).

MONTILLOR, C. B.; BERNAYS, E. A.; CORNELIUS, M. L. 1991. Responses of two hymenopteran predators to surface chemistry of their prey: significance for an alkaloid-sequestering caterpillar. Journal of Chemical Ecology, 17: 391-399.

MÜLLER, C.; AGERBIRK, N.; OLSEN, C.E.; BOEVE, J. L.; SCHAFFNER, U.; BRAKEFIELD, P. M. 2001. Sequestration of host plant glucosinolates in the defensive hemolymph of the sawfly Athalia rosae. Journal of Chemical Ecology, 27: 2505-2516.

MÜLLER, C.; BRAKEFIELD, P. M. 2003. Analysis of a chemical defense in sawfly larvae: easy bleeding targets predatory wasps in late summer. Journal of Chemical Ecology, 29: 2683-2694.

NISHIDA, R. 2002. Sequestration of defensive substances from plants by Lepidoptera. Annual Review of Entomology, 47: 57-92.

PARADISE, C.J.; STAMP, N. E. 1993. Episodes of unpalatable prey reduce consumption and growth of juvenile praying mantids. Journal of Insect Behavior, 6: 155-166.

RENWICK, J. A. A.; LOPEZ, K. 1999. Experience-based food consumption by larvae of Pieris rapae: addiction to glucosinolates? Entomologia Experimentalis et Applicata, 91: 51-58.

ROESSINGH, P.; HORA, K. H.; FUNG, S. Y.; PELTENBURG, A.; MENKEN, S. B. J. 2000. Host acceptance behaviour of the small ermine moth Yponomeuta cagnagellus: larvae and adults use different stimuli. Chemoecology, 10: 41-47.

SANTOS, G. P.; ZANUNCIO, J. C.; ZANUNCIO, T. V. 1996. Pragas do Eucalipto. Informe Agropecuário, 9: 63-71.

SANTOS, G. P.; ZANUNCIO, T. V.; ZANUNCIO, J. C. 2000. Desenvolvimento de Thyrinteina arnobia Stoll (Lepidoptera: Geometridae) em folhas de Eucalyptus urophylla e Psidium guajava. Anais da Sociedade Entomológica do Brasil, 29: 13-22.
SCHENK, D.; BACHER, S. 2002. Functional response of a generalist insect predator to one of its prey species in the field. Journal of Animal Ecology, 71: 524-531.

SGRILLO, R. B. 1982. A distribuição de Weibull como modelo de sobrevivência de insetos. Ecossistema, Espírito Santo do Pinhal, 7: 9-13.

STADLER, E. 2000. Secondary sulphur metabolites influencing herbivorous insects. In BRUNOLD, S. Sulfur nutrition and sulphur assimilation in higher plants. Paul Haupt, Bern, Switzerland, p. 187-202.

SZNAJDER, B.; HARVEY, J. A. 2003. Second and third trophic level effects of differences in plant species reflect dietary specialisation of herbivores and their endoparasitoids. Entomologia Experimentalis et Applicata, 109: 73-82.

VLIEGER, L.; BRAKEFIELD, P. M.; MÜLLER, C. 2004. Effectiveness of the defence mechanism of the turnip sawfly, Athalia rosae (Hymenoptera: Tenthredinidae), against predation by lizards. Bulletin of Entomological Research, 94: 283-289.

VINSON, S. B. 1999. Parasitoid manipulation as a plant defense strategy. Annals of the Entomological Society of America, 92: 812-828.

WEISER, L. A.; STAMP, N. E. 1998. Combined effects of allelochemicals, prey availability and supplemental plant material on growth of a generalist insect predator. Entomologia Experimentalis et Applicata, 87: 181-189.

WIEDENMANN, R. N.; LEGASPI, J. C.; O'NEIL, R. J. 1996. Impact of prey density and facultative plant feeding on the life history of the predator Podisus maculiventris (Heteroptera: Pentatomidae). In ALOMAR, O.; WIEDENMANN, R.N. Zoophytophagous Heteroptera: Implications for life history and integrated pest management. Annals of the Entomological Society of America, p. 94-118.

WINK, M.; GRIMM, C.; KOSCHMIEDER, C.; SPORER, F.; BERGEOT, O. 2000. Sequestration of phorbolesters by the aposematically coloured bug Pachycoris klugii (Heteroptera: Scutelleridae) feeding on Jatropha (Euphorbiaceae). Chemoecology, 10: 179-184.

ZANUNCIO, J. C.; GARCIA, J. F.; SANTOS, G. P.; ZANUNCIO, T. V.; NASCIMENTO, E.C. 1990a. Biologia e consumo foliar de lagartas de Euselasia apisaon (Dalman, 1823) (Lepidoptera:Riodinidae) em Eucalyptus spp. Revista Árvore, 14: 45-54.

ZANUNCIO, J. C.; FAGUNDES, M.; ANJOS, N.; ZANUNCIO, T. V.; CAPITANI, L. R. 1990b. Levantamento e flutuação populacional de lepidópteros associados à eucaliptocultura: V- Região de Belo Oriente, Minas Gerais, junho de 1986 a maio de 1987. Revista Árvore, 14: 35-44.

ZANUNCIO, J. C.; SANTOS, G. P.; BATISTA, L. G.; GASPERAZZO. W. L. 1993. Alguns aspectos da biologia de Dirphia rosacordis (Lepidoptera: Saturnidae) em folhas de eucalipto. Revista Árvore, 36: 112-117.

ZANUNCIO, J. C.; DO NASCIMENTO, E. C.; GARCIA, J. F.; ZANUNCIO, T. V. 1994a. Major lepidopterous defoliators of eucalypt in southeast Brazil. Forest Ecology and Management, 65: 53-63.

ZANUNCIO, J. C.; LEITE, J.E.M.; SANTOS, G. P.; NASCIMENTO, C. E. 1994b. Nova metodologia para criação em laboratório de hemípteros predadores. Revista Ceres, 41: 88-93.

ZANUNCIO, T. V.; ZANUNCIO, J. C.; TORRES, J. B.; LARANJEIRO, A. J. 1995. Biologia de Euselasia hygenius (Lepidoptera; Riodinidae) e seu consumo foliar em Eucalyptus urophylla. Revista Brasileira de Entomologia, 3: 487-492. 